\title{
Características espacio-tiempo de la sismicidad superficial en la Región Sur del Perú durante el período de 1976 a 2005
}

\author{
Space Features-Time Seismicity Superficial in Southern Peru \\ during the Period from 1976 to 2005
}

\author{
Víctor Aguilar Puruhuaya*, Roberto Kosaka Masuno** \\ http://dx.doi.org/10.21503/CienciayDesarrollo.2006.v7.05
}

\section{RESUMEN}

El objetivo de este estudio consiste en identificar la presencia de asperezas y agrupamientos de sismos a partir de la distribución espacio-tiempo de sismicidad superficial en el Sur del Perú, entre las coordenadas $13^{\circ}-19^{\circ}$ Sur y $69^{\circ}-78^{\circ}$ Oeste. (Ver figura 1.)

En este estudio se ha utilizado el Catálogo de National Earthquake Information Center (NEIC) (1976 - 2005), con 696 sismos de foco superficial $(\mathrm{h}<70 \mathrm{~km}$ ) y magnitud mínima de $4,5 \mathrm{Mb}$. La restricción de datos se produce en su nivel máximo de profundidad, debido a que se estima que a la profundidad de $60 \mathrm{~km}$ se encuentra el límite de contacto entre las superficies de las placas de Nasca y Sudamericana, dentro del proceso de subducción (Heras, 2002). Se eliminaron sismos menores a 4,5 Mb de magnitud, porque al no ser registrados en un gran número de las estaciones de la red mundial, sus parámetros hipocentrales no son de buena calidad. Asimismo, se ha procedido a eliminar las réplicas de sismos grandes, asumiendo una distribución temporal de 20 días.

Palabras clave: sismicidad, subducción, Placa de Nasca, Placa Sudamericana.

\section{ABSTRACT}

The aim of this study is to identify the presence of roughness and / or clusters of earthquakes from space-time distribution of seismicity surface occurred in southern Peru between coordinates $13^{\circ}-19^{\circ}$ South and $69^{\circ}-78^{\circ}$ West .

This study has used the catalogue National Earthquake Information Center (NEIC) (1976 - 2005), with 696 earthquakes focus surface $(\mathrm{h}<70 \mathrm{~km}$.) And magnitude of at least $4.5 \mathrm{Mb}$. The restriction data, is when its maximum depth because it is estimated that the depth of $60 \mathrm{~km}$. is the limited contact between the surfaces of the Nasca and South American plates in the process of subduction (Heras, 2002). It eliminated minor earthquakes of magnitude $4.5 \mathrm{Mb}$, because not being registered in a large number of stations of the global network, its parameters hipocentrales are not of good quality. Also, it proceeded to remove replicas of large earthquakes assuming a temporal distribution of 20 days.

Key words: seismicity, subduction, Nasca Plate, South American Plate.

\footnotetext{
* Instituto Geofísico. UNSA.

${ }^{* *}$ Universidad Alas Peruanas.
} 


\section{INTRODUCCIÓN}

La actividad sísmica del Perú tiene su origen en el proceso de subducción de la placa de Nasca bajo la Sudamericana, presente de norte a sur en su borde occidental, con una velocidad promedio del orden 7-8 cm/año (De Mets et al., 1990; Norabuena et al., 1999). Este proceso es responsable de la ocurrencia de los sismos de mayor magnitud que se hayan producido frente a la línea de costa (Dorbath et al., 1990; Tavera y Buforn, 2001), todos asociados al contacto sismogénico interplacas. Estos sismos son frecuentes en el tiempo, y en un año es posible registrar la ocurrencia de hasta 60 sismos, con magnitudes $m b>4,5$, y, en general, todos sentidos en las localidades cercanas a la línea de costa.

Estos movimientos se producen sobre la superficie de contacto de placas, la misma que presenta una longitud del orden de $2000 \mathrm{~km}$ en el borde occidental del Perú, y que para generar sismos debe hacerlo comprometiendo áreas pequeñas (de $200 \mathrm{~km}$ en el caso de sismos con magnitudes de 7,0 M, y áreas menores para sismos de pequeña magnitud). La acumulación de sismos en dichas áreas puede ser explicada con el modelo de Asperezas, el mismo que está basado en la idea de los gaps o lagunas sísmicas presentes en las zonas de subducción y fue desarrollado por Kanamori (1981). En estas zonas, los terremotos grandes tienden a repetirse en el mismo lugar después de un determinado período de tiempo. En este modelo, se supone la existencia de una gran falla, en donde se presentan zonas rotas y otras no (superficie de contacto de placas). Los esfuerzos iniciales no son homogéneos, debido al hecho de haberse presentado terremotos antes del sismo actual y los esfuerzos han sido liberados. El nuevo terremoto comienza donde no ha habido terremotos con anterioridad, y sobre todo donde se ha producido una gran acumulación de esfuerzos.

\section{MATERIAL Y MÉTODOS}

El análisis de la sismicidad se realiza sobre mapas de distribución espacial para el período que va de 1976 a 2005. El análisis mejora al analizar la distribución de los sismos en función del espacio-tiempo en mapas con períodos de 5 años. (Ver figura 2.)

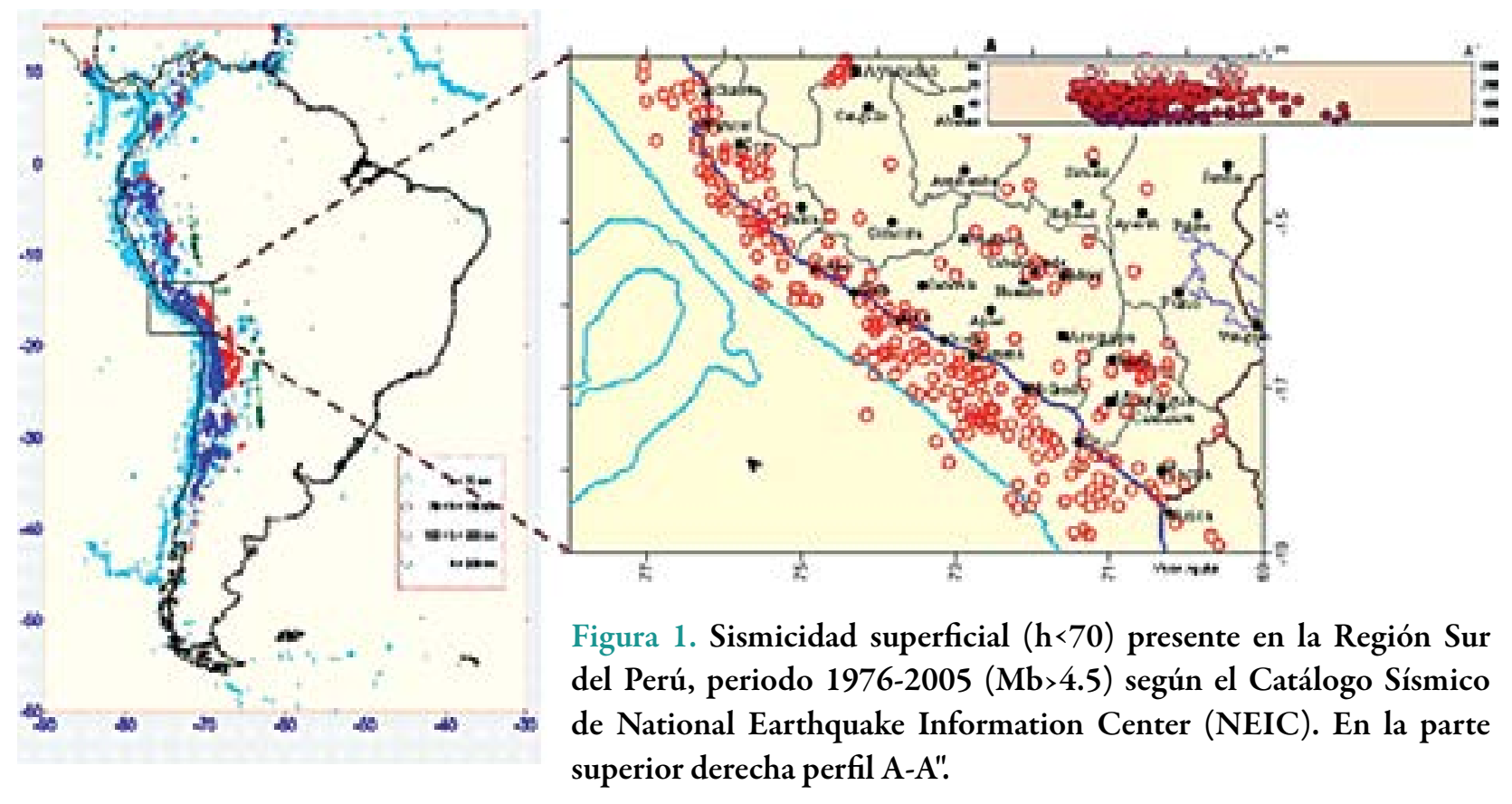




\section{RESULTADOS}

Según la figura 1, la mayor concentración de sismos se encuentra entre la fosa y la línea de costa, mientras que en el interior, la sismicidad disminuye considerablemente, aunque existen ciertos agrupamientos que podrían estar asociados a la presencia de algunas fallas geológicas.

En la figura 2a, se muestra la actividad sísmica para el período 1976 - 1980. Existen concentraciones de sismos entre las latitudes $-14,5^{\circ}$ y $-16^{\circ}$, y otras frente a la localidad de Camaná y Ocoña, cercanos al epicentro del sismo de magnitud 6,8 M ocurrido en el año 1979 (estrella). Se observa, asimismo, una disminución de la actividad sísmica entre la fosa y la línea de costa, entre las latitudes $-13^{\circ}$ y $-19^{\circ}$.

En la figura $2 b$, se muestra la actividad sísmica en el período que va de 1981 a 1985 . Los sismos se muestran concentrados entre las latitudes $-16^{\circ}$ y $-17,5^{\circ}$, y otros al SO de Ilo y al oeste de Ayacucho. Se observa, asimismo, que no hay actividad sísmica entre la fosa y la línea de costa, entre las latitudes $-13^{\circ}$ y $-19^{\circ}$. Es probable que en este caso se esté acumulando energía que provocará próximamente un sismo mayor.

En la figura 2c, se muestra la actividad sísmica del período 1986 - 1990. Sobresalen cuatro agrupamientos de sismos: uno frente a la ciudad de Nasca y tres frente a Mollendo, Ilo y al oeste de la ciudad de Tacna. Se observa también la ausencia de sismos entre la fosa y la línea de costa, entre las latitudes $-13^{\circ}$ y $-19^{\circ}$. Probablemente se esté acumulando también en este caso energía para un próximo sismo mayor.

En la figura 2d, aparece la actividad sísmica en el período 1991 - 1995. Se identifica la presencia de un agrupamiento de sismos entre las latitudes $-14,5^{\circ} \mathrm{y}-15,5^{\circ}$, y de otro en el continente, cerca de la localidad de Colca (sismos Maca 1991 y Sepina 1992). Se observa, asimismo, una disminución de sismos entre la fosa y la línea de costa, entre las latitudes $-13^{\circ}$ y $-19^{\circ}$. Es probable que se esté acumulando energía que será liberada en un próximo sismo mayor.

En la figura 2e, se presenta la actividad sísmica en el período 1996 - 2000. Se puede notar una acumulación de sismos entre las latitudes $-16^{\circ} \mathrm{y}$ $-17,5^{\circ}$, y otra al SO de Chincha. Otra acumulación de sismos está presente cerca del epicentro de sismos de Nasca de 1996 ( $\mathrm{Mw}=7,7$, estrella verde) y al SO de Mollendo. Se observa también la ausencia de sismos entre la fosa y la línea de costa, entre las latitudes $-13^{\circ}$ y $-19^{\circ}$.

En la figura $2 f$, se muestra la actividad sísmica en el período 2001 - 2005. Se verifican concentraciones de sismos entre las latitudes $-16^{\circ}$ y $-18^{\circ}$, asociadas al sismo del 23 de junio del año $2001(\mathrm{Mw}=8,2)$. Un agrupamiento de sismos se hace notorio al oeste de la ciudad de Tacna, y otro en el interior del continente por la reactivación del sistema de fallas presente en Moquegua. También se visualiza un alineamiento de sismos al oeste de Ica. Se observa una disminución de la actividad sísmica entre la fosa y la línea de costa, entre las latitudes $-13^{\circ}$ y $-16^{\circ}$ y entre las latitudes $-18^{\circ}$ y $-19^{\circ}$. Con estrellas verdes se señala la ubicación de los terremotos de 1868 y 2001, y las réplicas mayores, con estrellas de color azul.

\section{CONCLUSIONES}

El análisis de la distribución espacial de la sismicidad superficial ha permitido identificar la presencia de asperezas con diferentes dimensiones y longitudes, todas ellas provocadas por los sismos ocurridos entre los años 1996 y 2001. En estas áreas se produjeron sismos en el pasado; por lo tanto, ocurrirán también en el futuro y posiblemente tengan las mismas características en cuanto a la ubicación hipocentral y tamaño (magnitud). La historia sísmica indica que en la región Sur del Perú los sismos de magnitud ele- 

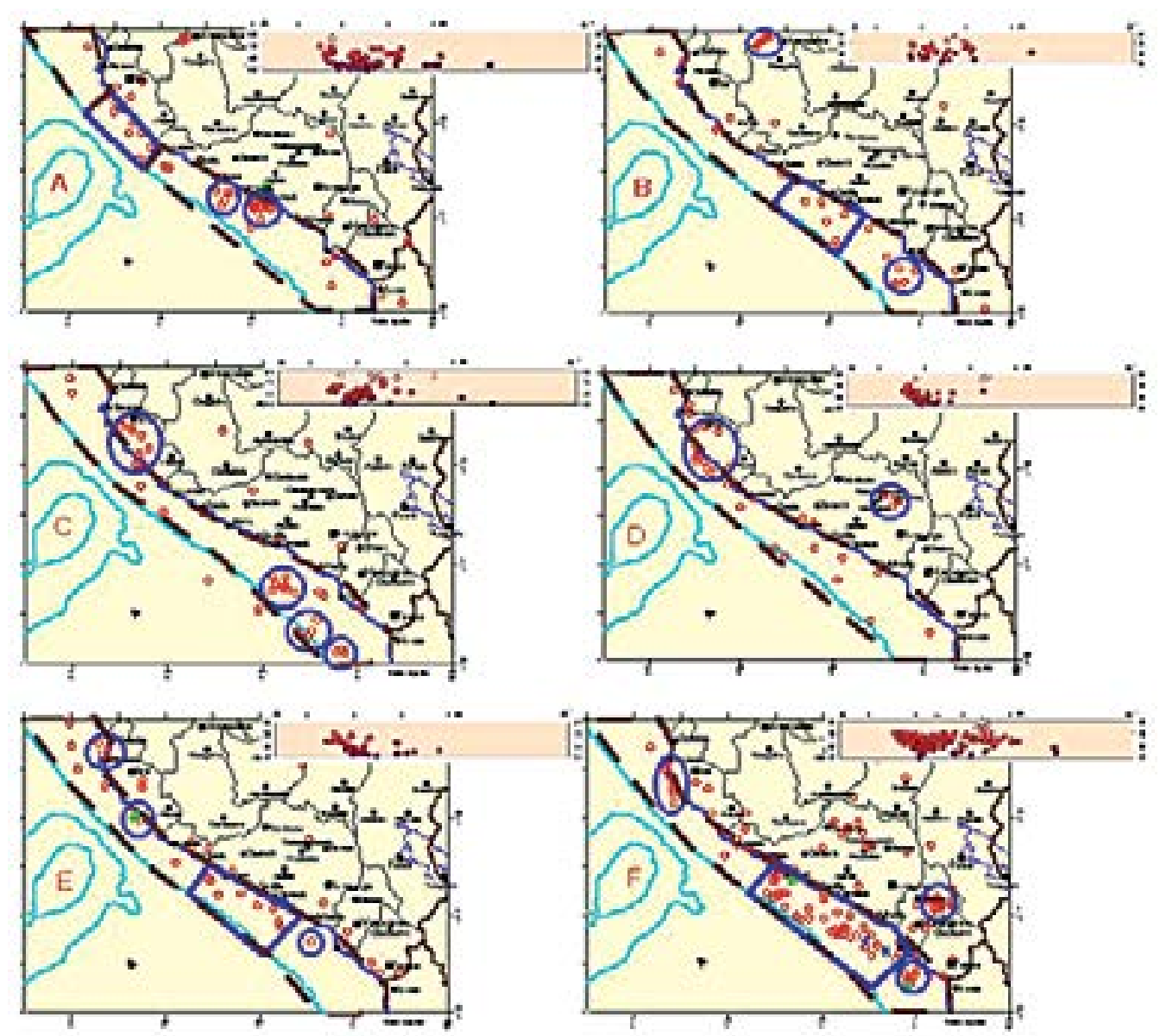

Figura 2. Mapas de actividad sísmica en la zona, de 1976 a 2005.

vada produjeron longitudes de ruptura del orden de 300 a $500 \mathrm{~km}$. Así, la intensidad del terremoto de $1868(\mathrm{Mw}=9,0)$, el mayor ocurrido en esta zona, no se ha repetido desde hace 138 años. Esto hace que dicha región sea identificada como la mayor zona de laguna sísmica, con una longitud de ruptura del orden de $500 \mathrm{~km}$, la misma que se vio afectada parcialmente por el terremoto del 2001, que tuvo una longitud de ruptura de 370 $\mathrm{km}$. Actualmente, no existe un método o técnica que permita predecir con exactitud el lugar, intensidad y la fecha de un sismo en una determinada región, debido a que cada sismo presenta características físicas y tectónicas particulares y muy complejas en su origen.

Los resultados obtenidos en este estudio son un ejemplo de la importante deformación super- ficial presente en áreas pequeñas. Estas deformaciones superficiales son provocadas por sismos de magnitud moderada, con la consecuente ocurrencia de un número importante de réplicas durante períodos de tiempo muy cortos.

Desde el año 1976 se observa una disminución de la actividad sísmica entre las latitudes que van de los de $13^{\circ}$ a los $16^{\circ}$ de Latitud Sur, y luego ocurre el terremoto de Nasca de 1996. La ausencia de actividad sísmica, desde el año de 1976, entre las latitudes de $16^{\circ}$ y $19^{\circ}$ de Latitud Sur señala la acumulación de energía que posteriormente es liberada en el terremoto del 2001. En las figuras $2 a, 2 b, 2 c, 2 d$ y $2 e$ se observa un agrupamiento de sismos de magnitud moderada al SO de la localidad de Mollendo, en los años 1997 y 2000, antes del terremoto. 
A fin de analizar la distribución de los sismos en función de la profundidad de sus focos, se han elaborado secciones verticales para cada período de tiempo, según la línea $\mathrm{A}-\mathrm{A}^{\prime}$. Estas secciones son perpendiculares a la fosa. Durante el período que va de 1976 a 2005, la sismicidad en la región se distribuye en profundidad, siguiendo una línea con pendiente próxima a los $30^{\circ}$, hasta una profundidad de $60 \mathrm{~km}$ aproximadamente, con patrones de sismicidad muy similares a los observados por otros autores (Tavera, 1998).

\section{REFERENCIAS BIBLIOGRÁFICAS}

1. Aguilar et al. "Características espacio-tiempo de la sismicidad superficial en la región Sur del Perú durante el período de 1976 a 2003". Trabajo presentado en el XII Congreso Peruano de Geología (Lima, Perú). 2004.

2. Barazangi M. \& Isaac L., 1976. "Spacial distribution of earthquakes and subduccion of the Nazca Plate beneath South America”. Geology, 4, 686-692, 1976.
3. Catálogo de National Earthquake Information Center (NEIC) (1976 - 2005)

4. CNDG. El terremoto de la Región Sur del Perú del 23 de junio. H. Tavera (Editor). Instituto Geofísico del Perú, 422 pp., 2002.

5. DeMets et al. Current plate motions. Geophys. J. Int., 101, 425-478, 1990.

6. Dorbath et al. "Assessment in the size of large and great historical earthquake in Peru". Bull. Seism. Soc. Am., 80, 551-576, 1990.

7. Heras H. Análisis de la distribución del valor de "b" en la zona de subducción del Perú. Tesis de grado. Universidad Nacional de San Agustín, Arequipa, 2002.

8. Kanamori H. "The nature of seismology patterns before large earthquake", In Earthquake Predictionan International Review; Maurice Ewing Ser., Ed. D.W., Simpsn P. G. Richards, 4, 1-19, Washintong D.G. Am. Gefhys. Union, 1981.

9. Norabuena et al. "Deceleration Nasca-South America and Nasca-Pacific plate motions". Geophys. Res. Lett., 26, 3405-3, 1999. 